\title{
PLEDs and NCSE: To Split or Lump them Together?
}

\section{Keni Ravish Rajiv, Deepak Menon and Ashalatha Radhakrishnan*}

Department of Neurology, R. Madhavan Nayar Center for Comprehensive Epilepsy

Care, Sree Chitra Tirunal Institute For Medical Sciences and Technology,

Trivandrum, Kerala, India

*Corresponding Author: Ashalatha Radhakrishnan, Professor of Neurology and Epileptologist, Department of Neurology, R. Madhavan Nayar Center for Comprehensive Epilepsy Care, Sree Chitra Tirunal Institute of Medical Sciences and Technology, Trivandrum, Kerala, India.
Received: July 26, 2020

Published: August 31, 2020

(C) All rights are reserved by

Radhakrishnan., et al.

\begin{abstract}
Periodic lateralised epileptiform discharges are electrophysiological biomarkers of acute and sometimes chronic brain injury. A major controversy through the years has been whether PLEDs are truly ictal or just interictal or post-ictal epiphenomenon, and whether these discharges warrant any treatment. In this review, we aimed to critically analyze this issue of whether to actively treat or wait and watch PLEDs in various situations. We conclude that the etiology of PLEDs, depth of encephalopathy and presence of clinical seizures is associated with poor outcome rather than presence of PLEDs per se. Continuous EEG monitoring is warranted to recognise ictal or high risk interictal EEG patterns akin to NCSE before treating aggressively with antiepileptic drugs.
\end{abstract}

Keywords: PLEDs; BiPLEDs; Seizures; NCSE; Outcome

\section{Abbreviations}

AED: Anti-Epileptic Drug; biPLEDs: Bilateral/Bihemispheric Independent PLEDs; cEEG: Continuous EEG; NCSE: Non Convulsive Status Epilepticus; PLEDs: Periodic Lateralised Epileptiform Discharges; ePLEDs: Emergent PLEDs; SPECT: Single Photon Emission Computed Tomography

\section{Introduction}

Periodic lateralised epileptiform discharges (PLEDs) are the surrogate markers in EEG mostly of an emergent (ePLEDs) and at times long-standing, underlying structural or functional brain injury. Many early studies have shown an increased mortality of around $40-50 \%$ in patients with ePLEDs [1-4]. But a closer review of some of these data reveals rather than the mere presence of ePLEDs, the underlying etiology has a greater bearing on the outcome. With potentially reversible etiologies like sub-therapeutic anticonvulsant levels, alcohol or barbiturate withdrawal, hypertensive encephalopathy and hyperosmolar coma having a good outcome and potentially lethal ones like acute major stroke or anoxia having a poor outcome with higher morbidity and mortal- ity [1]. With the advent of continuous EEG (cEEG) in the emergent setting, the clinical and prognostic significance of these discharges and their evolution from and to electrographic seizures have been recognized in more detail. Thus, a major controversy through the years has been whether PLEDs are truly ictal or just inter-ictal or post-ictal epiphenomenon, being the finger prints of the criminal rather than the criminal itself and whether these discharges warrant any treatment [5]. In this review, we would like to critically analyze this issue of whether to actively treat or wait and watch PLEDs in various situations.

\section{Review of Literature}

Search strategy included free-text and MeSH terms "PLEDs" AND “NCSE" AND "treatment", in LILACS, EMBASE, PubMed, and The Cochrane Library. We are emphasizing only the important studies which have debated or raised concerns on the core issue pertaining to this review as to treat or not to treat PLEDs.

Borderland between PLEDs and non-convulsive status epilepticus (NCSE)

Recently with the revised definitions of NCSE (Modified Salz- 
burg Consensus Criteria), the distinction of ePLEDs from NCSE and whether they both mandate treatment has again become a matter of debate or rather a controversy [6]. The diagnosis of NCSE as per this revised definition and consensus is "any condition amounting to any alteration in behavior or mental process from baseline with continuous epileptic discharges in EEG". Any ictal patterns with a typical spatiotemporal evolution or epileptiform discharges faster than $2.5 \mathrm{~Hz}$ in a comatose patient reflect NCSE and should be treated. So, the distinction is only in the frequency of discharges, distinction between NCSE and PLEDs is that PLEDs are considered when the frequency of discharges is less than $2.5 \mathrm{~Hz}$. But as noted earlier by Reiher., et al. when these so called slower frequency discharges are associated with faster rhythmic discharges lasting at least 2 seconds (PLEDs proper getting converted to PLEDs plus (PLEDS those with rhythmic discharges associated with PLEDs lasting more than 2 seconds), it is considered a fore-runner of electrographic and thereby clinical seizures [7]. This remains unchanged in Salzburg criteria as well. Thus, whether patients with pure NCSE versus those having PLEDs with coma which is considered equivalent to NCSE (especially since differentiating coma from encephalopathy is rather difficult and confusing) are to be treated needs to be debated. In this setting the authors propose the following algorithmic approach in acutely ill patients who have ePLEDs (excluding chronic PLEDs).

PLEDs are just EEG signatures: do not need active treatment

We looked into the available literature considering PLEDs as just an indication of the acute neurological dysfunction in the EEG following an acute insult to the brain parenchyma, where they emphasize that they need urgent attention and very active management as in ongoing seizures only in few settings.

In authors' own experience with long term follow-up of 1948 patients who underwent emergent EEG, those with ePLEDs were associated with significant morbidity and mortality [8]. However, the etiology of ePLEDs, impairment of consciousness and seizures at presentation predicted poor outcome than PLEDs per say. There was also a trend for higher functional disability in patients with BiPLEDs (bihemispheric/bilateral independent PLEDs) compared to PLEDs. Other similar studies using cEEG have noted findings such as NCSE, abnormal background activity in EEG, PLEDs plus and generalised periodic epileptiform discharges as highly predictive of poor outcome [9]. In a recent retrospective study of 40 patients with ePLEDs, 35 being treated and 5 untreated, mortality was unchanged with or without treatment of patients with PLEDs on cEEG [10]. Patients with chronic PLEDs have been reported and ictal discharges in the EEGs are distinct from the PLEDs and dur- ing ictus the PLEDs disappear, suggesting PLEDs to be an interictal phenomenon [11].

PLEDs are EEG signatures of ictal activity: they need active treatment

Garzon., et al. [12], prospectively followed up 55 patients with SE having 254 EEG recordings, unequivocally noted that PLEDs was one of the four ictal correlates of seizures. At the same time, they also noted that PLEDs/BiPLEDs were not associated with time course, outcome or prognosis. They demonstrated that, although PLEDs were not always associated with seizures and SE, they are unequivocally an ictal pattern. This was reinforced by application of single photon emission computed tomography(SPECT) in PLEDs which showed that areas of high cerebral blood flow coincided with location of PLEDs in $94 \%$ cases and that the same cleared once PLEDs disappeared, pointing to a possible epileptic nature of these discharges [13]. Interestingly, in a patient with a right parietal parenchymal metastasis from pulmonary cancer, hypoperfusion of the lesion and the adjacent cortex was also observed during both quiescence and appearance of PLEDs using SPECT [14]. Similarly, cerebral hypoperfusion on SPECT was also noted in a patient with cerebral lupus and PLEDs [15]. Indicating that reduction in regional cerebral blood flow on SPECT implies brain functional impairment.

Approach to a patient with PLEDs: Diagnosis, differentiating from NCSE and deciding on treatment

Step 1: Identifying the etiology and clinical setting of PLEDs [1$4,8,9,11]$

In most studies, the etiology of PLEDs remains the single important predictor of outcome with non-neoplastic and reversible causes having a better outcome. Not only the outcome, the risk of progression to seizures is highest in acute structural brain lesions, for example stroke, traumatic brain injury, acute encephalitic process etc; previous epilepsy, drug default with seizures, prolonged obtundation without significant improvement and acute worsening should be considered as pointers favoring treatment of PLEDs.

\section{Step 2: Clinical phenomenology $[5,6,8,9]$}

The clinician should be on the lookout for subtle neurological features of ictal manifestations. The presence of altered state of consciousness, also called "confusional state" or "consciousness disturbance" and more specific complaints including nystagmus retractorius, cortical blindness, depression, apraxia, aphasia, amnesia, hemianopia, hemiparesis, gaze preference or deviation, nystagmus, dysphasia and speech impediment have been described in association with PLEDs. However, the presence of these features would point more in favor of NCSE with ongoing brain injury due to epileptogenic activity and should alert the physician for treatment. 
Step 3: PLEDs morphology and evolution (Role of cEEG) $[6,7,9]$

The importance of the EEG characteristics is particularly true especially when deciding whether the PLEDs, rather than the etiology of ePLEDs is the cause of encephalopathy and thus mandates antiepileptic drugs. As discussed earlier, a cEEG monitoring is warranted to recognise EEG patterns such as i) clear incremental or decremental pattern and a spatiotemporal evolution would strongly favor ongoing ictal activity. ii) presence of PLEDs plus and BiPLEDs are clear markers which announce high risk for further progression to seizure.

\section{Step 4: Response to treatment}

It seems reasonable starting or maintaining a conventional anti-epileptic drug (AED) in all patients with ePLEDs without escalating treatment unless clear ictal electrographic or clinical se- miology is observed [16]. Given the availability of medications that are easily administered with relatively low toxicity, a conventional AED at a relatively low dose can be administered in patients with ePLEDs especially when uncertain as to its evolution. In patients having ictal or high risk inter ictal EEG patterns for NCSE as discussed above, it is worth giving a trial of IV benzodiazepines (Figure 1). The clinical improvement is defined as better performance in one of the five domains [17]: (i) "say your surname", (ii) "repeat $1,2,3$ ", (iii) "raise your arms", (iv) patient opens eyes to i-iii, and (v) patient looks at the examiner in response to i-iii. If no response, repeat the procedure after strong tactile stimuli on both sides of the body. EEG improvement would be reduction of these discharges to less than $10 \%$ of the epoch. If there is a significant improvement it warrants initiation or stepping-up of AEDs, whereas lack of significant improvement warrants cEEG monitoring and evaluation for other causes of encephalopathy.



Figure 1: Algorithm for approach to a patient with ePLEDs: diagnosis, differentiating from NCSE and its management.

AED:Anti epileptic drug; biPLEDs: Bihemispheric PLEDs; cEEG: Continuous EEG; GTCS: Generalised Tonic-Clonic Seizure; PLEDs: Periodic Lateralised Epileptiform Discharges; TBI: Traumatic Brain Injury. 


\section{Conclusion}

PLEDs are electrophysiological biomarkers of acute and sometimes chronic brain injury. Whether PLEDs are truly ictal or just inter ictal or post ictal epiphenomenon, is debatable. The etiology of PLEDs, depth of encephalopathy and presence of clinical seizures is associated with poor outcomes rather than presence of PLEDs per se. cEEG monitoring is warranted to recognise ictal or highrisk inter-ictal EEG patterns for NCSE before treating aggressively with AEDs. A clear line cannot be drawn as to when ePLEDs qualifies for NCSE is difficult, though a cEEG monitoring with a video recording and close observation of the patient and EEG would be a timely and reasonable approach at this juncture with the available evidence.

\section{Acknowledgements}

This research did not receive any grant from the public, commercial, or not for profit sector funding agencies.

\section{Disclosure}

None of the authors has any conflicts of interest to disclose. We confirm that we have read the Journal's position on issues involved in ethical publication and affirm that this report is consistent with those guidelines.

\section{Bibliography}

1. Walsh JM and Brenner RP. "Periodic lateralized epileptiform discharges long-term outcome in adults". Epilepsia 28 (1987): 533-536.

2. De la Paz D and Brenner RP. "Bilateral independent periodic lateralized epileptiform discharges. Clinical significance". Archives of Neurology 38 (1981): 713-715.

3. Kuroiwa Y and Celesia GG. "Clinical significance of periodic EEG patterns". Archives of Neurology 37 (1980): 15-20.

4. Porecha HP and Reilly EL. "Clinical Correlations of Periodic Lateralized Epileptiform Discharges". Clinical Electroencephalography 8.4 (1977): 191-202.

5. Kaplan PW. "EEG criteria for nonconvulsive status epilepticus". Epilepsia 48 (2007): 39-41.

6. Leitinger M., et al. "Salzburg Consensus Criteria for Non-Convulsive Status Epilepticus--approach to clinical application". Epilepsy and Behavior 49 (2015): 158-163.

7. Reiher J., et al. "Periodic lateralized epileptiform discharges with transitional rhythmic discharges: association with seizures". Electroencephalography and Clinical Neurophysiology 78.1 (1991): 12-17.
8. Kate MP., et al. "Long-term outcome and prognosis of patients with emergent periodic lateralized epileptiform discharges (ePLEDs)". Seizure 21.6 (2012): 450-456.

9. Rai V., et al. "Continuous EEG predictors of outcome in patients with altered sensorium". Seizure 22.8 (2013): 656-661.

10. Ahmad Solaiman A., et al. "When Should We Treat Periodic Lateralized Epileptiform Discharges (PLEDS) (P5.061)". Neurology 82.10 (2014).

11. Chatrian GE., et al. "The significance of periodic lateralized epileptiform discharges in EEG: an electrographic, clinical and pathological study". Electroencephalography and Clinical Neurophysiology 17 (1964): 177-193.

12. Garzon E., et al. "Serial EEG during human status epilepticus: evidence for PLED as an ictal pattern". Neurology 57.7 (2001): 1175-1183.

13. Assal F., et al. "SPECT in periodic lateralized epileptiform discharges (PLEDs): a form of partial status epilepticus?" Seizure 10 (2001): 260-265.

14. Hisada K., et al. "Magnetoencephalographic analysis of periodic lateralized epileptiform discharges (PLEDs)". Clinical Neurophysiology 111 (2000): 122-127.

15. Aye SM., et al. "Periodic lateralized epileptiform discharges (PLEDs) in cerebral lupus correlated with white-matter lesions in brain MRI and reduced cerebral blood flow in SPECT". Lupus 22 (2013): 510-514.

16. Lee JW. "EEG in the ICU. What should one treat, what not?" Epileptologie 29 (2012): 210-217.

17. Trinka E and Leitinger M. "Which EEG patterns in coma are nonconvulsive status epilepticus?" Epilepsy and Behavior 49 (2015): 203-222.

\section{Assets from publication with us}

- Prompt Acknowledgement after receiving the article

- Thorough Double blinded peer review

- Rapid Publication

- Issue of Publication Certificate

- High visibility of your Published work

Website: www.actascientific.com/

Submit Article: www.actascientific.com/submission.php Emaill us: editor@actascientific.com

Contact us: +919182824667 